\title{
Regioselective Introduction of Homoallylic Amine Moiety to Quinolines: Preparation of Reissert Compound Followed by In-Mediated Allylation of Nitrile
}

\author{
Sung Hwan Kim, Sangku Lee, ${ }^{\dagger}$ Yu Mi Kim, and Jae Nyoung Kim* \\ Department of Chemistry and Institute of Basic Science, Chonnam National University, Gwangju 500-757, Korea \\ *E-mail:kimjn@chonnam.ac.kr \\ ${ }^{\dagger}$ Natural Medicine Research Center, KRIBB, Daejeon 305-806, Korea \\ Received September 4, 2010, Accepted September 27, 2010
}

Key Words: Indium, Homoallylic amine, Quinolines, Reissert compounds, Allylation

Homoallylic amines are valuable building blocks in organic synthesis as precursors for $\beta$-amino acids and many heterocycles. ${ }^{1-3}$ In addition, the structural motif is present in numerous biologically important substances. ${ }^{3}$

During our recent studies on the reaction of allylindium reagents and nitrile moiety-containing substrates ${ }^{4}$ we found that the imine intermediate, generated in situ by the reaction of a nitrile and allylindium reagents, could be quenched by a suitable electrophilic quencher in the same molecule to form a cyclic compound. ${ }^{4}$ Inter- and intramolecular quenching of the imine intermediate has been known in limited cases. ${ }^{5}$

Based on the previous results ${ }^{4,5}$ we reasoned out that a regioselective introduction of a homoallylic amine moiety at the 2-position of quinoline could be accomplished by a simple two-step procedure, as shown in Scheme 1. The first step is a synthesis of Reissert compound of quinoline ${ }^{6}$ and the next is an In-mediated allylation of nitrile moiety of the Reissert compound. ${ }^{4,7}$ In the intermediate stage, an intramolecular transfer of benzoyl group would be possible because the dihydroquinoline residue is a good leaving group and the $N$-benzoylimine intermediate could be converted into the homoallylic amine by a consecutive $1,3-\mathrm{H}$ shift (see, path-a in Scheme 2).

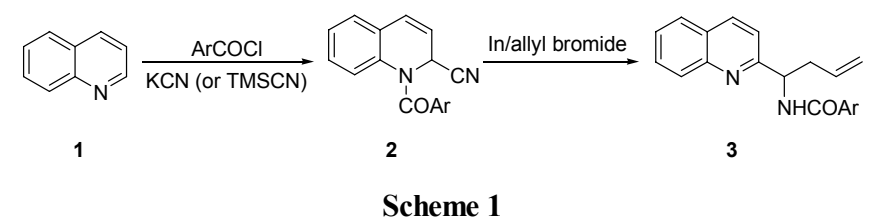

In order to examine the feasibility of our assumption, a representative Reissert compound 2a was prepared according to the reported method. ${ }^{6 f}$ The reaction of $\mathbf{2 a}$ and allyl bromide was carried out in THF in the presence of indium metal at refluxing temperature for $20 \mathrm{~min}$. To our delight, desired compound 3a was obtained in good yield $(70 \%)$ along with a trace amount of imidazo $[1,5-a]$ quinoline $4 \mathbf{a}(6 \%){ }^{8}$. The reaction mechanism can be postulated as shown in Scheme 2. The reaction of $2 \mathbf{a}$ and allylindium reagents produced an imine intermediate (I), which produced $N$-benzoylimine intermediate (II) by following the path-a. The dihydroquinoline moiety acts as a leaving group to form (II). Successive 1,3-H transfers of (II) produced a homoallylamine derivative 3a. Compound 4a was formed in a trace amount via the dehydrative cyclization (path-b). ${ }^{8}$

Encouraged by the results, various Reissert compounds $\mathbf{2 b - e}$ were prepared in high yields $(85-89 \%)$ and the reactions with allylindium (or methallylindium) reagents were carried out. As shown in Table 1, the corresponding homoallylic amine derivatives $\mathbf{3 b}$-h were obtained in reasonable yields $(50-79 \%)$. The reactions with methallyl bromide (entries 6-8) afforded the corresponding products in higher yields than the cases of allyl bromide (entries 1-5). However, the reactions of $\mathbf{2} \mathbf{a}$ with benzyl bromide or crotyl bromide did not produce the corresponding amines in reasonable yields under the same reaction conditions. The reactions showed very sluggish reactivity and produced many intractable side products.

It is interesting to note that the reaction of a Reissert compound 2f, derived from isoquinoline, did not produce the corresponding homoallylic amine $\mathbf{5}$ under the same reaction conditions,

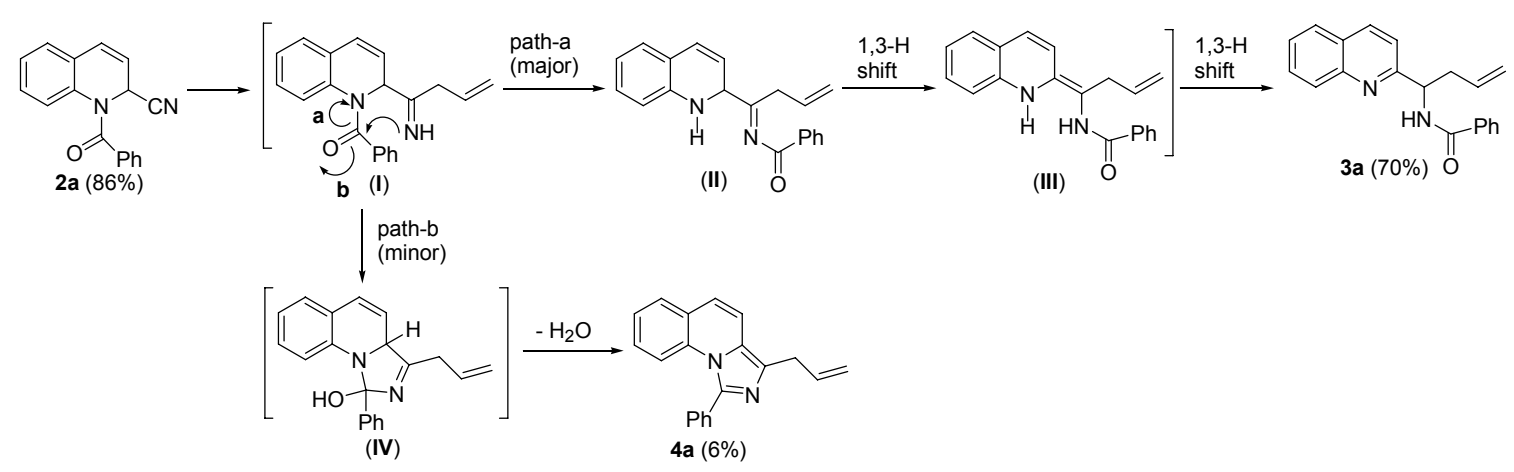

Scheme 2 


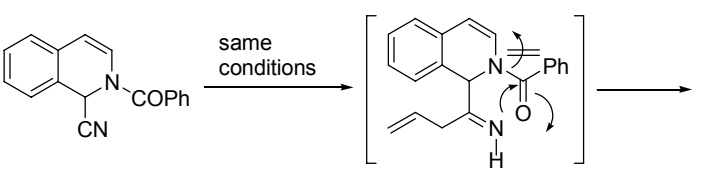

$2 f$<smiles>C=CCc1nc(-c2ccccc2)n2ccc3ccccc3c12</smiles>

$6(17 \%)$<smiles>C=CCC(NC(=O)c1ccccc1)c1nccc2ccccc12</smiles>

5 (not formed)

Scheme 3

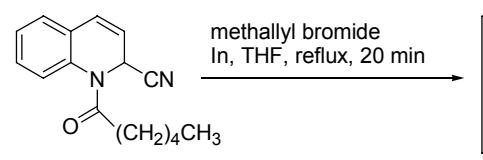

$2 g$

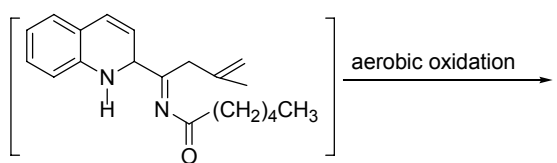

(V)

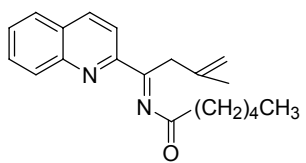

$7(52 \%)$

Scheme 4

Table 1. In-mediated synthesis of $N$-aroylhomoallylic amines

Entry

${ }^{a}$ Prepared by $\mathrm{PhCOCl} / \mathrm{KCN} / \mathrm{CH}_{2} \mathrm{Cl}_{2} / \mathrm{H}_{2} \mathrm{O}$ or $\mathrm{PhCOCl} / \mathrm{TMSCN} / \mathrm{CH}_{2} \mathrm{Cl}_{2}$ as reported. ${ }^{6}{ }^{b}$ Conditions: Substrate (1.0 equiv), allyl bromide (3.0 equiv), In (1.5 equiv), THF, reflux, $20 \mathrm{~min}^{c} \mathrm{Ar}$ is $p$-tolyl. ${ }^{d}$ Methallyl bromide was used.

as shown in Scheme 3. Instead, a imidazo[5,1-a]isoquinoline derivative 6 was isolated in low yield (17\%). The failure might be due to the less leaving ability of dihydroisoquinoline moiety (Scheme 3) than the dihydroquinoline moiety in the case of qui- noline (Scheme 2).

The reaction of $N$-hexanoyl derivative $\mathbf{2 g}$ did not produce the corresponding product, quite unexpectedly (Scheme 4 ). We isolated $N$-hexanoylimine derivative 7 in moderate yield (52\%). This compound might be formed via an aerobic oxidation of the corresponding $N$-hexanoylimine intermediate $(\mathbf{V})$. The reason for this unusual reactivity of $\mathbf{2} \mathbf{g}$ is unclear at this stage.

In summary, an efficient two-step procedure was developed for the introduction of a homoallylic amine moiety at the 2-position of various quinolines. ${ }^{9}$ The reaction involved a synthesis of Reissert compound and a subsequent In-mediated allylation of nitrile.

\section{Experimental Section}

Preparation of starting materials. The Reissert compounds were prepared according to the reported procedures. ${ }^{6 f-i}$ As a cyanide source $\mathrm{KCN}$ was used in most cases; however TMSCN was used for the preparation of $\mathbf{2} \mathbf{c}^{6 \mathrm{~g}}$ and $\mathbf{2 g}$. The spectroscopic data of unknown compound $\mathbf{2} \mathbf{g}$ are as follows.

Compound 2g: 65\%; colorless oil; IR (film) 2234, 1659, $1493,1335 \mathrm{~cm}^{-1} ;{ }^{1} \mathrm{H} \mathrm{NMR}\left(\mathrm{CDCl}_{3}, 300 \mathrm{MHz}\right) \delta 0.84(\mathrm{t}, J=6.9$ $\mathrm{Hz}, 3 \mathrm{H}), 1.19-1.35$ (m, 4H), 1.53-1.73 (m, 2H), 2.32-2.64 (m, $2 \mathrm{H}), 6.08(\mathrm{dd}, J=9.0$ and $6.0 \mathrm{~Hz}, 1 \mathrm{H}), 6.51(\mathrm{~d}, J=6.0 \mathrm{~Hz}, 1 \mathrm{H})$, $6.76(\mathrm{~d}, J=9.0 \mathrm{~Hz}, 1 \mathrm{H}), 7.24-7.38(\mathrm{~m}, 4 \mathrm{H}) ;{ }^{13} \mathrm{C} \mathrm{NMR}\left(\mathrm{CDCl}_{3}\right.$, $75 \mathrm{MHz}) \delta 14.11,22.54,25.53,31.49,33.89,40.52,116.16$, 122.24, 124.85, 127.07, 127.37, 127.81, 128.94, 129.62, 134.15, 173.02; ESIMS $m / z 255\left(\mathrm{M}^{+}+\mathrm{H}\right)$. Anal. Calcd for $\mathrm{C}_{16} \mathrm{H}_{18} \mathrm{~N}_{2} \mathrm{O}$ : C, 75.56; H, 7.13; N, 11.01. Found: C, 75.79; H, 7.33; N, 10.89 .

Typical procedure for the synthesis of $3 \mathrm{a}$. A stirred mixture of $2 \mathbf{a}(130 \mathrm{mg}, 0.5 \mathrm{mmol})$, allyl bromide (181 mg, $1.5 \mathrm{mmol})$, and indium $(86 \mathrm{mg}, 0.75 \mathrm{mmol})$ in THF $(2 \mathrm{~mL})$ was heated to reflux for $20 \mathrm{~min}$. After the usual aqueous workup and column chromatographic purification process (hexanes $/ \mathrm{CH}_{2} \mathrm{Cl}_{2} / \mathrm{EtOAc}$, 7:2:1) compound 3a was isolated as a pale yellow solid (106 mg, $70 \%$ ) along with $\mathbf{4 a}(9 \mathrm{mg}, 6 \%)$. Other compounds $\mathbf{3 b}$-h were synthesized similarly, and the spectroscopic data of $\mathbf{3 a}, \mathbf{4 a}, \mathbf{3} \mathbf{b}-\mathbf{h}$, 6, and $\mathbf{7}$ are as follows.

Compound 3a: 70\%; pale yellow solid, mp 116 - $117^{\circ} \mathrm{C}$; IR (KBr) $3325,1643,1506,1484 \mathrm{~cm}^{-1} ;{ }^{1} \mathrm{H} \mathrm{NMR}\left(\mathrm{CDCl}_{3}, 300 \mathrm{MHz}\right)$ $\delta 2.76-2.84(\mathrm{~m}, 1 \mathrm{H}), 2.88-2.97(\mathrm{~m}, 1 \mathrm{H}), 5.00-5.07(\mathrm{~m}, 2 \mathrm{H}), 5.52$ $(\mathrm{dt}, J=6.9$ and $6.0 \mathrm{~Hz}, 1 \mathrm{H}), 5.67-5.81(\mathrm{~m}, 1 \mathrm{H}), 7.39(\mathrm{~d}, J=8.4$ 
$\mathrm{Hz}, 1 \mathrm{H}), 7.44-7.56(\mathrm{~m}, 4 \mathrm{H}), 7.72(\mathrm{ddd}, J=8.4,6.9$ and $1.5 \mathrm{~Hz}$, $1 \mathrm{H}), 7.81(\mathrm{dd}, J=8.1$ and $0.9 \mathrm{~Hz}, 1 \mathrm{H}), 7.91-7.94(\mathrm{~m}, 2 \mathrm{H}), 8.09$ $(\mathrm{d}, J=8.4 \mathrm{~Hz}, 1 \mathrm{H}), 8.11(\mathrm{~d}, J=6.9 \mathrm{~Hz}, \mathrm{NH}), 8.14(\mathrm{~d}, J=8.4 \mathrm{~Hz}$, $1 \mathrm{H}) ;{ }^{13} \mathrm{CNMR}\left(\mathrm{CDCl}_{3}, 75 \mathrm{MHz}\right) \delta 40.40,53.86,118.34,120.15$, $126.39,127.05,127.35,127.62,128.50,129.01,129.62,131.36$, 133.35, 134.81, 136.69, 147.19, 159.20, 166.66; ESIMS m/z 303 $\left(\mathrm{M}^{+}+\mathrm{H}\right)$. Anal. Calcd for $\mathrm{C}_{20} \mathrm{H}_{18} \mathrm{~N}_{2} \mathrm{O}: \mathrm{C}, 79.44 ; \mathrm{H}, 6.00 ; \mathrm{N}, 9.26$. Found: C, 79.51; H, 6.28; N, 9.12.

Compound 4a: 6\%; pale yellow oil; IR (film) 1661, 1483, $1454,1371 \mathrm{~cm}^{-1} ;{ }^{1} \mathrm{H} \mathrm{NMR}\left(\mathrm{CDCl}_{3}, 300 \mathrm{MHz}\right) \delta 3.71(\mathrm{dt}, J=6.3$ and $1.5 \mathrm{~Hz}, 2 \mathrm{H}), 5.09-5.25(\mathrm{~m}, 2 \mathrm{H}), 6.07-6.20(\mathrm{~m}, 1 \mathrm{H}), 6.95$ $(\mathrm{d}, J=9.3 \mathrm{~Hz}, 1 \mathrm{H}), 7.14(\mathrm{ddd}, J=8.7,7.2$ and $1.2 \mathrm{~Hz}, 1 \mathrm{H}), 7.30$ $(\mathrm{dd}, J=7.2$ and $1.2 \mathrm{~Hz}, 1 \mathrm{H}), 7.32(\mathrm{~d}, J=9.3 \mathrm{~Hz}, 1 \mathrm{H}), 7.46-$ $7.53(\mathrm{~m}, 4 \mathrm{H}), 7.59$ (dd, $J=7.8$ and $1.5 \mathrm{~Hz}, 1 \mathrm{H}), 7.62-7.65$ $(\mathrm{m}, 2 \mathrm{H}) ;{ }^{13} \mathrm{C} \mathrm{NMR}\left(\mathrm{CDCl}_{3}, 75 \mathrm{MHz}\right) \delta 32.54,115.91,116.99$, $117.62,120.37,125.26,125.96,127.10,127.42,128.75,128.79$, 129.02, 129.46, 129.93, 132.65, 134.02, 136.61, 141.37; ESIMS $m / z 285\left(\mathrm{M}^{+}+\mathrm{H}\right)$.

Compound 3b: $61 \%$; pale yellow solid, mp $127-128^{\circ} \mathrm{C}$; IR (KBr) 3341, 1645, 1599, 1514, $1483 \mathrm{~cm}^{-1}$; ${ }^{\mathrm{H}} \mathrm{NMR}\left(\mathrm{CDCl}_{3}\right.$, $300 \mathrm{MHz}) \delta 2.58(\mathrm{~s}, 3 \mathrm{H}), 2.79-2.89(\mathrm{~m}, 1 \mathrm{H}), 2.92-3.01(\mathrm{~m}, 1 \mathrm{H})$, $5.03-5.11(\mathrm{~m}, 2 \mathrm{H}), 5.55(\mathrm{dt}, J=6.9$ and $6.0 \mathrm{~Hz}, 1 \mathrm{H}), 5.71-5.85$ $(\mathrm{m}, 1 \mathrm{H}), 7.39(\mathrm{~d}, J=8.4 \mathrm{~Hz}, 1 \mathrm{H}), 7.48-7.61(\mathrm{~m}, 5 \mathrm{H}), 7.96-7.99$ $(\mathrm{m}, 2 \mathrm{H}), 8.03(\mathrm{~d}, J=8.4 \mathrm{~Hz}, 1 \mathrm{H}), 8.09(\mathrm{~d}, J=8.4 \mathrm{~Hz}, 1 \mathrm{H}), 8.17$ $(\mathrm{d}, J=6.9 \mathrm{~Hz}, \mathrm{NH}) ;{ }^{13} \mathrm{C} \mathrm{NMR}\left(\mathrm{CDCl}_{3}, 75 \mathrm{MHz}\right) \delta 21.46,40.40$, 53.79, 118.25, 120.10, 126.47, 127.05, 127.37, 128.48, 128.66, 131.31, 131.84, 133.43, 134.87, 136.01, 136.24, 145.75, 158.21, 166.63; ESIMS m/z $317\left(\mathrm{M}^{+}+\mathrm{H}\right)$.

Compound 3c: $68 \%$; pale yellow solid, mp $125-126^{\circ} \mathrm{C}$; IR (KBr) 3323, 1641, 1599, 1514, $1487 \mathrm{~cm}^{-1}$; ${ }^{1} \mathrm{H} \mathrm{NMR}\left(\mathrm{CDCl}_{3}, 300\right.$ MHz) $\delta 2.75-2.83(\mathrm{~m}, 1 \mathrm{H}), 2.86-2.93(\mathrm{~m}, 1 \mathrm{H}), 5.00-5.07$ (m, $2 \mathrm{H}), 5.52(\mathrm{dt}, J=6.9$ and $6.0 \mathrm{~Hz}, 1 \mathrm{H}), 5.68-5.77(\mathrm{~m}, 1 \mathrm{H}), 7.40$ $(\mathrm{d}, J=8.7 \mathrm{~Hz}, 1 \mathrm{H}), 7.43-7.51(\mathrm{~m}, 3 \mathrm{H}), 7.62(\mathrm{dd}, J=9.0$ and $2.4 \mathrm{~Hz}, 1 \mathrm{H}), 7.77(\mathrm{~d}, J=2.4 \mathrm{~Hz}, 1 \mathrm{H}), 7.90-7.93(\mathrm{~m}, 2 \mathrm{H}), 7.99$ $(\mathrm{d}, J=6.9 \mathrm{~Hz}, \mathrm{NH}), 8.00(\mathrm{~d}, J=9.0 \mathrm{~Hz}, 1 \mathrm{H}), 8.02(\mathrm{~d}, J=8.4$ $\mathrm{Hz}, 1 \mathrm{H}) ;{ }^{13} \mathrm{C} \mathrm{NMR}\left(\mathrm{CDCl}_{3}, 75 \mathrm{MHz}\right) \delta 40.19,53,81,118.48$, $120.98,126.23,127.01,127.85,128.49,130.44,130.55,131.41$, 132.03, 133.16, 134.61, 135.72, 145.52, 159.67, 166.66; ESIMS $m / z 337\left(\mathrm{M}^{+}+\mathrm{H}\right), 339\left(\mathrm{M}^{+}+2+\mathrm{H}\right)$. Anal. Calcd for $\mathrm{C}_{20} \mathrm{H}_{17} \mathrm{ClN}_{2} \mathrm{O}$ : C, 71.32; H, 5.09; N, 8.32. Found: C, 71.17; H, 5.34; N, 8.21.

Compound 3d: $50 \%$; pale yellow solid, mp $135-136^{\circ} \mathrm{C}$; IR (KBr) 3335, 1645, 1601, 1503, $1483 \mathrm{~cm}^{-1} ;{ }^{1} \mathrm{H} \mathrm{NMR}\left(\mathrm{CDCl}_{3}, 300\right.$ MHz) $\delta 2.74-2.94$ (m, 2H), 3.92 (s, 3H), 4.99-5.06 (m, 2H), 5.48 $(\mathrm{dt}, J=7.2$ and $6.0 \mathrm{~Hz}, 1 \mathrm{H}), 5.66-5.80(\mathrm{~m}, 1 \mathrm{H}), 7.07(\mathrm{~d}, J=2.7$ $\mathrm{Hz}, 1 \mathrm{H}), 7.34(\mathrm{~d}, J=8.4 \mathrm{~Hz}, 1 \mathrm{H}), 7.37(\mathrm{dd}, J=9.0$ and $2.7 \mathrm{~Hz}$, 1H), 7.43-7.51 (m, 3H), 7.90-7.93 (m, 2H), 7.98 (d, $J=9.6 \mathrm{~Hz}$, 1H), $8.02(\mathrm{~d}, J=8.4 \mathrm{~Hz}, 1 \mathrm{H}), 8.07$ (d, $J=7.2 \mathrm{~Hz}, \mathrm{NH}) ;{ }^{13} \mathrm{C} \mathrm{NMR}$ $\left(\mathrm{CDCl}_{3}, 75 \mathrm{MHz}\right) \delta 40.45,53.70,55.50,105.12,118.24,120.43$, 122.26, 127.04, 128.31, 128.49, 130.34, 131.33, 133.49, 134.81, 135.46, 143.24, 156.67, 157.67 166.66; ESIMS $m / z 333\left(\mathrm{M}^{+}+\mathrm{H}\right)$.

Compound 3e: $65 \%$; pale yellow solid, mp $138-139^{\circ} \mathrm{C}$; IR (KBr) 3335, 1643, 1612, 1524, $1491 \mathrm{~cm}^{-1}$; ${ }^{1} \mathrm{H} \mathrm{NMR}\left(\mathrm{CDCl}_{3}\right.$, $300 \mathrm{MHz}) \delta 2.40(\mathrm{~s}, 3 \mathrm{H}), 2.74-2.84(\mathrm{~m}, 1 \mathrm{H}), 2.86-2.95(\mathrm{~m}, 1 \mathrm{H})$, 4.98-5.06 (m, 2H), $5.51(\mathrm{dt}, J=7.2$ and $6.0 \mathrm{~Hz}, 1 \mathrm{H}), 5.67-5.80$ (m, 1H), $7.26(\mathrm{~d}, J=7.8 \mathrm{~Hz}, 2 \mathrm{H}), 7.38(\mathrm{~d}, J=8.4 \mathrm{~Hz}, 1 \mathrm{H}), 7.52$ (ddd, $J=8.1,6.9$ and $1.2 \mathrm{~Hz}, 1 \mathrm{H}$ ), 7.71 (ddd, $J=8.4,6.9$ and 1.5 $\mathrm{Hz}, 1 \mathrm{H}), 7.80(\mathrm{dd}, J=8.1$ and $1.2 \mathrm{~Hz}, 1 \mathrm{H}), 7.82(\mathrm{~d}, J=7.8 \mathrm{~Hz}$, $2 \mathrm{H}), 8.06(\mathrm{~d}, J=7.2 \mathrm{~Hz}, \mathrm{NH}), 8.08(\mathrm{~d}, J=8.1 \mathrm{~Hz}, 1 \mathrm{H}), 8.12$ (d,
$J=8.7 \mathrm{~Hz}, 1 \mathrm{H}) ;{ }^{13} \mathrm{C} \mathrm{NMR}\left(\mathrm{CDCl}_{3}, 75 \mathrm{MHz}\right) \delta 21.74,40.72$, 54.18, 118.61, 120.52, 126.69, 127.39, 127.68, 127.94, 129.32, 129.49, 129.91, 132.27, 133.76, 137.00, 142.05, 147.53, 159.70, 166.98; ESIMS $m / z 317\left(\mathrm{M}^{+}+\mathrm{H}\right)$.

Compound 3f: $79 \%$; pale yellow solid, mp $107-108^{\circ} \mathrm{C}$; IR (KBr) 3223, 1639, 1614, 1533, $1501 \mathrm{~cm}^{-1} ;{ }^{1} \mathrm{H}$ NMR $\left(\mathrm{CDCl}_{3}\right.$, $300 \mathrm{MHz}) \delta 1.78(\mathrm{~s}, 3 \mathrm{H}), 2.76(\mathrm{dd}, J=6.9$ and $0.6 \mathrm{~Hz}, 2 \mathrm{H})$, 4.69-4.70 (m, 1H), 4.79-4.80 (m, 1H), $5.59(\mathrm{dt}, J=7.5$ and 6.9 $\mathrm{Hz}, 1 \mathrm{H}), 7.39$ (d, $J=8.4 \mathrm{~Hz}, 1 \mathrm{H}), 7.41-7.53$ (m, 4H), 7.70 (ddd, $J=8.4,6.9$ and $1.5 \mathrm{~Hz}, 1 \mathrm{H}), 7.79(\mathrm{dd}, J=8.1$ and $1.2 \mathrm{~Hz}$, 1H), $7.85(\mathrm{~d}, J=7.5 \mathrm{~Hz}, \mathrm{NH}), 7.88-7.91(\mathrm{~m}, 2 \mathrm{H}), 8.06-8.09(\mathrm{~m}$, $1 \mathrm{H}), 8.10(\mathrm{~d}, J=8.4 \mathrm{~Hz}, 1 \mathrm{H}) ;{ }^{13} \mathrm{C} \mathrm{NMR}\left(\mathrm{CDCl}_{3}, 75 \mathrm{MHz}\right) \delta$ 22.46, 44.72, 53.02, 114.24, 120.23, 126.24, 126.99, 127.32, $127.56,128.44,128.93,129.49,131.29,134.72,136.47,141.59$, 147.35, 160.04, 166.62; ESIMS $m / z 317\left(\mathrm{M}^{+}+\mathrm{H}\right)$. Anal. Calcd for $\mathrm{C}_{21} \mathrm{H}_{20} \mathrm{~N}_{2} \mathrm{O}$ : C, 79.72; H, 6.37; N, 8.85. Found: C, 79.66; $\mathrm{H}, 6.56 ; \mathrm{N}, 8.71$.

Compound 3g: $60 \%$; pale yellow solid, mp $129-130{ }^{\circ} \mathrm{C}$; IR (KBr) 3333, 1643, 1601, 1501, $1485 \mathrm{~cm}^{-1} ;{ }^{1} \mathrm{H}$ NMR $\left(\mathrm{CDCl}_{3}\right.$, $300 \mathrm{MHz}) \delta 1.77(\mathrm{~s}, 3 \mathrm{H}), 2.74(\mathrm{~d}, J=6.9 \mathrm{~Hz}, 2 \mathrm{H}), 3.92(\mathrm{~s}, 3 \mathrm{H})$, 4.68-4.69 (m, 1H), 4.78-4.79 (m, $1 \mathrm{H}), 5.55(\mathrm{dt}, J=7.5$ and 6.9 $\mathrm{Hz}, 1 \mathrm{H}), 7.06(\mathrm{~d}, J=2.7 \mathrm{~Hz}, 1 \mathrm{H}), 7.35(\mathrm{~d}, J=8.4 \mathrm{~Hz}, 1 \mathrm{H}), 7.36$ $(\mathrm{dd}, J=9.3$ and $2.7 \mathrm{~Hz}, 1 \mathrm{H}), 7.42-7.51(\mathrm{~m}, 3 \mathrm{H}), 7.80(\mathrm{~d}, J=$ $7.5 \mathrm{~Hz}, \mathrm{NH}), 7.87-7.91(\mathrm{~m}, 2 \mathrm{H}), 7.97$ (d, $J=9.3 \mathrm{~Hz}, 1 \mathrm{H}), 8.01$ $(\mathrm{d}, J=8.4 \mathrm{~Hz}, 1 \mathrm{H}) ;{ }^{13} \mathrm{C} \mathrm{NMR}\left(\mathrm{CDCl}_{3}, 75 \mathrm{MHz}\right) \delta 22.52,44.86$, 52.89, 55.50, 105.11, 114.21, 120.61, 122.24, 127.04, 128.34, $128.49,130.33,131.33,134.80,135.30,141.75,143.47,157.53$, 157.62, 166.65; ESIMS m/z $347\left(\mathrm{M}^{+}+\mathrm{H}\right)$.

Compound 3h: $76 \%$; pale yellow solid, mp $114-115^{\circ} \mathrm{C}$; IR (KBr) 3325, 1641, 1599, 1504, $1485 \mathrm{~cm}^{-1} ;{ }^{1} \mathrm{H}$ NMR $\left(\mathrm{CDCl}_{3}\right.$, $300 \mathrm{MHz}) \delta 1.78(\mathrm{~s}, 3 \mathrm{H}), 2.39(\mathrm{~s}, 3 \mathrm{H}), 2.76(\mathrm{dd}, J=6.9$ and 0.6 $\mathrm{Hz}, 2 \mathrm{H}), 4.68-4.69$ (m, 1H), 4.78-4.80 (m, 1H), 5.58 (dt, $J=$ 7.5 and $6.9 \mathrm{~Hz}, 1 \mathrm{H}), 7.23-7.26(\mathrm{~m}, 2 \mathrm{H}), 7.39(\mathrm{~d}, J=8.4 \mathrm{~Hz}, 1 \mathrm{H})$, $7.51(\mathrm{ddd}, J=8.1,6.9$ and $1.2 \mathrm{~Hz}, 1 \mathrm{H}), 7.70$ (ddd, $J=8.4,6.9$ and $1.5 \mathrm{~Hz}, 1 \mathrm{H}), 7.78-7.81(\mathrm{~m}, 4 \mathrm{H}), 8.06-8.09(\mathrm{~m}, 1 \mathrm{H}), 8.10$ $(\mathrm{d}, J=8.4 \mathrm{~Hz}, 1 \mathrm{H}) ;{ }^{13} \mathrm{C} \mathrm{NMR}\left(\mathrm{CDCl}_{3}, 75 \mathrm{MHz}\right) \delta 21.39,22.49$, 44.76, 53.02, 114.23, 120.34, 126.27, 127.03, 127.36, 127.60, 128.94, 129.13, 129.52, 131.87, 136.51, 141.68, 141.71, 147.40, 160.24, 166.63; ESIMS $m / z 331\left(\mathrm{M}^{+}+\mathrm{H}\right)$.

Compound 6: 17\%; pale yellow oil; IR (film) 1638, 1603, $1481,1462 \mathrm{~cm}^{-1} ;{ }^{1} \mathrm{H}$ NMR $\left(\mathrm{CDCl}_{3}, 300 \mathrm{MHz}\right) \delta 4.00(\mathrm{dt}, J=$ 5.7 and $1.8 \mathrm{~Hz}, 2 \mathrm{H}), 5.12-5.22(\mathrm{~m}, 2 \mathrm{H}), 6.13-6.26(\mathrm{~m}, 1 \mathrm{H})$, $6.72(\mathrm{~d}, J=7.2 \mathrm{~Hz}, 1 \mathrm{H}), 7.38(\mathrm{td}, J=7.2$ and $0.9 \mathrm{~Hz}, 1 \mathrm{H})$, 7.41-7.56 (m, 5H), 7.75-7.78 (m, 2H), $7.94(\mathrm{~d}, J=7.5 \mathrm{~Hz}, 1 \mathrm{H})$, $8.07(\mathrm{~d}, J=8.1 \mathrm{~Hz}, 1 \mathrm{H}) ;{ }^{13} \mathrm{C} \mathrm{NMR}\left(\mathrm{CDCl}_{3}, 75 \mathrm{MHz}\right) \delta 34.50$, $113.89,115.93,120.76,123.12,124.33,126.04,126.14,126.93$, 127.44, 128.28, 128.60, 128.88, 128.91, 129.98, 133.14, 135.09, 139.33; ESIMS $m / z 285\left(\mathrm{M}^{+}+\mathrm{H}\right)$.

Compound 7: 52\%; pale yellow oil; IR (film) 1651, 1626, $1477,1454,1385 \mathrm{~cm}^{-1}$; ${ }^{1} \mathrm{H}$ NMR $\left(\mathrm{CDCl}_{3}, 300 \mathrm{MHz}\right) \delta 0.93(\mathrm{t}$, $J=6.9 \mathrm{~Hz}, 3 \mathrm{H}), 1.35-1.52(\mathrm{~m}, 4 \mathrm{H}), 1.76(\mathrm{~s}, 3 \mathrm{H}), 1.87-1.98(\mathrm{~m}$, $2 \mathrm{H}), 3.33-3.38(\mathrm{~m}, 2 \mathrm{H}), 3.57(\mathrm{~s}, 2 \mathrm{H}), 4.77-4.78(\mathrm{~m}, 1 \mathrm{H}), 4.81-$ $4.82(\mathrm{~m}, 1 \mathrm{H}), 6.83(\mathrm{~d}, J=9.6 \mathrm{~Hz}, 1 \mathrm{H}), 7.22(\mathrm{~d}, J=9.6 \mathrm{H}, 1 \mathrm{H})$, 7.34 (ddd, $J=7.5,7.2$ and $1.5 \mathrm{~Hz}, 1 \mathrm{H}), 7.48$ (ddd, $J=8.7,7.2$ and $1.5 \mathrm{~Hz}, 1 \mathrm{H}), 7.59(\mathrm{dd}, J=7.5$ and $1.5 \mathrm{~Hz}, 1 \mathrm{H}), 8.06(\mathrm{~d}, J=$ $8.7 \mathrm{~Hz}, 1 \mathrm{H}) ;{ }^{13} \mathrm{C} \mathrm{NMR}\left(\mathrm{CDCl}_{3}, 75 \mathrm{MHz}\right) \delta 14.02,22.27,22.39$, $27.10,31.69,32.15,36.05,111.32,116.37,117.02,119.12$, $124.60,125.91,126.78,127.50,128.48,130.40,133.23,142.97$, 
144.29; ESIMS $m / z 309\left(\mathrm{M}^{+}+\mathrm{H}\right)$. Anal. Calcd for $\mathrm{C}_{20} \mathrm{H}_{24} \mathrm{~N}_{2} \mathrm{O}$ : C, 77.89; H, 7.84; N, 9.08 Found: C, 77.96; H, 7.56; N, 8.87.

Acknowledgments. This research was supported by the Basic Science Research Program through the National Research Foundation of Korea (NRF) funded by the Ministry of Education, Science and Technology (2010-0015675). Spectroscopic data were obtained from the Korea Basic Science Institute, Gwangju branch.

\section{References and Notes}

1. For the synthesis and applications of homoallylic amine derivatives, see: (a) Puentes, C. O.; Kouznetsov, V. J. Heterocyclic Chem. 2002, 39, 595-614. (b) Lou, S.; Moquist, P. N.; Schaus, S. E. J. Am. Chem. Soc. 2007, 129, 15398-15404. (c) Albano, V. G.; Gualandi, A.; Monari, M.; Savoia, D. J. Org. Chem. 2008, 73, 8376-8381. (d) Katritzky, A. R.; Ignatchenko, A. V.; Lang, H. J. Org. Chem. 1995, 60, 4002-4005. (e) Del Valle, J. R.; Goodman, M. J. Org. Chem. 2004, 69, 8946-8948. (f) White, J. D.; Hansen, J. D. J. Org. Chem. 2005, 70, 1963-1977. (g) Nicolaou, K. C.; Mitchell, H. J.; van Delft, F. L.; Rubsam, F.; Rodriguez, R. M. Angew. Chem. Int. Ed. 1998, 37, 1871-1874. (h) Robl, J. A.; Cimarusti, M. P.; Simpkins, L. M.; Brown, B.; Ryono, D. E.; Bird, J. E.; Asaad, M. M.; Schaeffer, T. R.; Trippodo, N. C. J. Med. Chem. 1996, 39, 494-502. (i) Vilaivan, T.; Winotapan, C.; Banphavichit, V.; Shinada, T.; Ohfune, Y. J. Org. Chem. 2005, 70, 3464-3471. (j) Rueping, M.; Antonchick, A. P. Angew. Chem. Int. Ed. 2008, 47, 10090-10093.

2. For the synthesis and applications of homoallylic amine derivatives attached directly at the heterocycles, see: (a) Singh, P.; Kaur, P.; Luxami, V.; Kaur, S.; Kumar, S. Bioorg. Med. Chem. 2007, 15, 2386-2395. (b) Kumar, S.; Kaur, P. Tetrahedron Lett. 2004, 45, 3413-3416. (c) Fiorelli, C.; Savoia, D. J. Org. Chem. 2007, 72, 6022-6028.

3. For the biologically important substances having a homoallylic amine moiety, see: (a) Suvire, F. D.; Sortino, M.; Kouznetsov, V. V.; Vargas M, L. Y.; Zacchino, S. A.; Cruz, U. M.; Enriz, R. D. Bioorg. Med. Chem. 2006, 14, 1851-1862. (b) Janjic, J. M.; Mu, Y.; Kendall, C.; Stephenson, C. R. J.; Balachandran, R.; Raccor, B. S.; Lu, Y.; Zhu, G.; Xie, W.; Wipf, P.; Day, B. W. Bioorg. Med. Chem. 2005, 13, 157-164. (c) Doherty, A. M.; Sircar, I.; Kornberg, B. E.; Quin, J., III.; Winters, T.; Kaltenbronn, J. S.; Taylor, M. D.; Batley, B. L.; Rapundalo, S. R.; Ryan, M. J.; Painchaud, C. A. J. Med. Chem. 1992, 35, 2-14.

4. For the intramolecular quenching of imine intermediate to form cyclic compounds, see: (a) Kim, S. H.; Lee, H. S.; Kim, K. H.; Kim, J. N. Tetrahedron Lett. 2009, 50, 1696-1698. (b) Kim, S. H.; Kim, S. H.; Lee, K. Y.; Kim, J. N. Tetrahedron Lett. 2009, 50, 5744-5747. (c) Kim, S. H.; Lee, H. S.; Kim, K. H.; Kim, J. N. Tetrahedron Lett. 2009, 50, 6476-6479. (d) Kim, S. H.; Kim, S. H.; Kim, K. H.; Kim, J. N. Tetrahedron Lett. 2010, 51, 860-862. (e) Kim, S. H.; Kim, S. H.; Kim, T. H.; Kim, J. N. Tetrahedron Lett. 2010, 51 , 2774-2777.

5. For the in-situ quenching of an indium intermediate, see: (a) Du, Z.; Li, Y.; Wang, F.; Zhou, W.; Wang, J.-X. Tetrahedron Lett. 2010, 51, 1745-1747. (b) Lombardo, M.; Pasi, F.; Trombini, C. Eur. J. Org. Chem. 2006, 3061-3063. (c) Kim, S. H.; Kim, Y. M.; Kim, J. N. Bull. Korean Chem. Soc. 2010, 31, 2351-2356.

6. For the synthesis of Reissert compounds, see: (a) Takamura, M.; Funabashi, K.; Kanai, M.; Shibasaki, M. J. Am. Chem. Soc. 2000, 122, 6327-6328. (b) Takamura, M.; Funabashi, K.; Kanai, M.; Shibasaki, M. J. Am. Chem. Soc. 2001, 123, 6801-6808. (c) Yadav, J. S.; Reddy, B. V. S.; Srinivas, M.; Sathaiah, K. Tetrahedron Lett. 2005, 46, 3489-3492. (d) Popp, F. D.; Kant, J. Heterocycles 1985, 23, 2193-2195. (e) Lizarraga, E.; Zabaleta, C.; Palop, J. A. Thermochim. Acta 2005, 427, 171-174. (f) Gibson, H. W.; Guilani, B. J. Org. Chem. 1990, 55, 4226-4229. (g) Elliott, I. W., Jr. J. Am. Chem. Soc. 1955, 77, 4408-4409. (h) Wolf, A. P.; McEwen, W. E.; Glazier, R. H. J. Am. Chem. Soc. 1956, 78, 861-868. (i) Popp, F. D.; Blount, W.; Melvin, P. J. Org. Chem. 1961, 26, 4930-4932.

7. For the general review on indium-mediated reactions, see: (a) Auge, J.; Lubin-Germain, N.; Uziel, J. Synthesis 2007, 1739-1764. (b) Li, C.-J.; Chan, T.-H. Tetrahedron 1999, 55, 11149-11176. (c) Kim, S. H.; Lee, H. S.; Kim, K. H.; Kim, S. H.; Kim, J. N. Tetrahedron 2010, 66, 7065-7076 and further references cited therein.

8. For the synthesis of similar compounds, see: (a) Fuchs, C.; Bender, C.; Ziemer, B.; Liebscher, J. J. Heterocyclic Chem. 2008, 45, 16511658. (b) Davey, D.; Erhardt, P. W.; Lumma, W. C., Jr.; Wiggins, J.; Sullivan, M.; Pang, D.; Cantor, E. J. Med. Chem. 1987, 30, 1337-1342. (c) Iwao, M.; Kuraishi, T. J. Heterocyclic Chem. 1979, 16, 689-698. (d) Kant, J. J. Heterocyclic Chem. 1990, 27, 21292132.

9. Deprotection of $N$-benzoyl group of $\mathbf{3} \mathbf{a}$ was easily conducted in aqueous $\mathrm{EtOH}$ in the presence of conc- $\mathrm{HCl}$ (reflux, $10 \mathrm{~h}$ ) in $86 \%$, and the spectroscopic data are as follows: pale yellow oil; IR (film) $3407,3358,1618,1599,1503,1379 \mathrm{~cm}^{-1} ;{ }^{1} \mathrm{H} \mathrm{NMR}\left(\mathrm{CDCl}_{3}, 300\right.$ MHz) $\delta 2.46-2.56(\mathrm{~m}, 1 \mathrm{H}), 2.64-2.73(\mathrm{~m}, 1 \mathrm{H}), 3.40(\mathrm{~s}, 2 \mathrm{H}), 4.31$ $(\mathrm{dd}, J=7.8$ and $5.4 \mathrm{~Hz}, 1 \mathrm{H}), 5.06-5.16(\mathrm{~m}, 2 \mathrm{H}), 5.71-5.85(\mathrm{~m}, 1 \mathrm{H})$, $7.42(\mathrm{~d}, J=8.1 \mathrm{~Hz}, 1 \mathrm{H}), 7.49$ (ddd, $J=8.1,6.9$ and $1.2 \mathrm{~Hz}, 1 \mathrm{H}$ ), 7.67 (ddd, $J=8.4,6.9$ and $1.5 \mathrm{~Hz}, 1 \mathrm{H}), 7.77(\mathrm{dd}, J=8.1$ and $1.2 \mathrm{~Hz}$, $1 \mathrm{H}), 8.05(\mathrm{~d}, J=8.4 \mathrm{~Hz}, 1 \mathrm{H}), 8.08(\mathrm{~d}, J=8.4 \mathrm{~Hz}, 1 \mathrm{H})$; ESIMS $\mathrm{m} / \mathrm{z} 199\left(\mathrm{M}^{+}+\mathrm{H}\right)$. 